\title{
1D Phenomenological Modeling of Shape Memory and Pseudoelasticity in NiTi Alloys
}

\author{
Carmine Maletta and Franco Furgiuele \\ Additional information is available at the end of the chapter
}

http://dx.doi.org/10.5772/51283

\section{Introduction}

A one-dimensional phenomenological approach to simulate both the mechanical and functional properties in shape memory alloys (SMAs) is described in the following sections. In fact, shape-memory alloys exhibit unique mechanical and functional features, due to reversible transformations in crystal structure. In particular, on the macroscopic scale, SMAs are able to remember a geometrical shape and can return to that shape by activating the phase transition mechanisms. Many kinds of SMAs have been exploited in the last decades, such as the copper-zinc-aluminum ( $\mathrm{ZnCuAl})$, copper-aluminum-nickel (CuAlNi), nickel-manganese-gallium (NiMnGa), nickel-titanium (NiTi), and other ones made by alloying zinc, copper, gold, iron, etc. Among these alloys the near equiatomic NiTi binary system shows the most exploitable characteristics due to the high stress and strain recovery capabilities associated with their functional properties, namely pseudoelastic effect (PE) and shape memory effect (SME). These properties are due to a reversible solid state phase transformation between a parent phase (austenite) and a product phase (martensite), the so called thermoelastic martensitic transformation (TMT), that can be activated either by temperature (Thermally Induced Martensite, TIM), or by applied stress (Stress Induced Martensite, SIM) [1]. Due to these features NiTi alloys are currently used in an increasing number of applications in many fields of engineering [2], for the realization of smart sensors and actuators, joining devices, hydraulic and pneumatic valves, release/separation systems, consumer applications and commercial gadgets. However, thanks to their good mechanical properties and biocompatibility the most important applications of NiTi alloys are in the field of medicine, where pseudoelasticity is mainly exploited for the realization of several components, such as cardiovascular stent, embolic protection filters, orthopedic components, orthodontic wires, micro surgical and endoscopic devices. As a direct consequence of their interesting features NiTi alloys have attracted the interest of scientific and engineering community in the last years. However, despite the increasing interest and the efforts of many researchers to better understand these unusual mechanisms, the use of NiTi alloys is currently 
limited to high-value applications (i.e. medical devices, MEMS, etc.), due to the high cost of the raw material as well as to the complex component manufacturing; in fact, an accurate control of the processing parameters must be carried out as the functional and mechanical properties of NiTi alloys are significantly affected by the thermo-mechanical loading history experienced during manufacturing [3-8]. On the other hand, the design of complex shaped NiTi-based components needs an accurate knowledge of the mechanical and functional response of the material, as well as how this evolves during subsequent thermo-mechanical processes. Within this context the use of numerical modeling techniques, to simulate both mechanical and functional behavior of SMAs, is of major concern and, consequently, many studies have been focused on this topic in the last few years $[9,10]$, with the aim to model the non-linear hysteretic behavior that describes the phase transformation, and the related functional properties. Some of these models are based on microscopic and mesoscopic approaches [10], where the thermo-mechanical behavior is modeled starting from molecular level and lattice level, respectively; other models are based on macroscopic approaches, where only phenomenological features of the SMAs are used [11-24]. In this field, some authors proposed one-dimensional models based on an assumed polynomial-free energy potential $[11,12]$ while other models are based on an assumed phase transformation kinetic and consider simple mathematical functions to describe the phase transformation behavior of the material [13-15]. These models are probably the most popular in the literature due to their phenomenological approaches, which allow easy developments without considering the underlying physics of the transformation kinetic. Furthermore, other models are based on the elastoplasticity theory [16-22] which are capable of describing the functional behavior of the material using plasticity concepts. Finally, some researchers used the Galerkin method to describe thermo-mechanical behaviors of shape memory alloys [23, 24]. More recently, a 1-D phenomenological approach to simulate both the shape memory effect [27-29] and pseudoelastic effect [30] in NiTi-based shape memory alloys has been developed and it is described in the following sections. In particular, the temperature-strain and stress-strain hysteretic behavior of SMAs, associated with the thermally induced and stress-induced phase transition mechanisms, are modeled from a phenomenological point of view, i.e. without considering the underlying physics of the problem, by using Prandtl-Ishlinksii hysteresis operators $[25,26]$. The main features of this approach is a simple implementation together with a good accuracy and efficiency in predicting the stress-strain hysteretic behavior of $1 \mathrm{D}$ components. Unfortunately, the one dimensional nature of the proposed model, represents one of the major drawback with respect to some of the pre-existing phenomenological models, which are based on more thermodynamically consistent frameworks and, consequently, are able to capture several behaviors of NiTi alloys, such as detailed stress-strain distribution in 2D and 3D components. However, the high computational efficiency of the proposed model allows its use for real time simulation and control o 1D SMA components. The parameters of the phenomenological model are identified by simple and efficient numerical procedures, starting from a set of experimentally measured hysteresis loops. The identification procedures have been developed in the commercial software package Matlab $b^{T M}$, while the computed parameters are used in Simulink ${ }^{T M}$ models, which are able to simulate the whole path dependent hysteretic behavior of the SMAs, i.e. for generic complete and incomplete stress-induced and/or thermally induced phase transition mechanisms. The models are also able to capture the hysteresis modifications due to complex loading conditions, i.e. they are able to predict the change of the transformation stresses and temperatures according to the 
Clausius-Clapeyron relation [1]. The unique thermo-mechanical features of SMAs are firstly illustrated in the following section 2, while the numerical approach is described in section 3 together with some case studies, involving both shape memory and pseudoelasticity, and the results show good accuracy and small computational time.

\section{Thermo-mechanical properties of NiTi alloys}

\subsection{Thermoelastic martensitic transformation}

Nickel-Titanium (NiTi) based shape memory alloys exhibit unique thermomechanical properties due to a reversible solid state phase transformation between a high temperature parent phase (B2 - austenite) and a low temperature product phase (B19' - martensite), the so called Thermoelastic Martensitic Transformation (TMT). In particular, TMT can be activated by a temperature variation (TIM, Thermally Induced Martensite) or by the application of an external mechanical load (SIM, Stress Induced Martensite), and it allows the crystal lattice structure to accommodate to the minimum energy state for a given temperature and/or stress. Figure 1 schematically shows the crystal structures of the two phases.
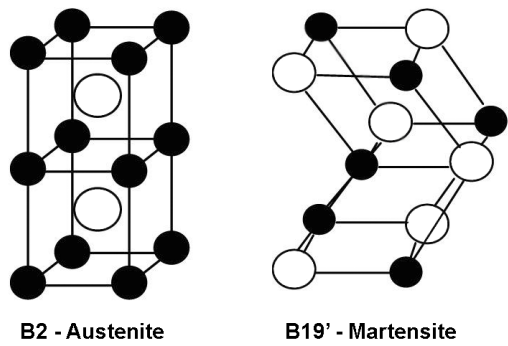

Figure 1. Schematic depiction of the lattice structures of austenite and martensite.

The austenitic phase is characterized by a Body Centered Cubic structure (BCC), with a nickel atom at the center of the crystallographic cube and titanium atoms at the cube's corners, while the lattice structure of the martensitic phase consists of a rhombus alignment with an atom at each of the rhombus corners. On the macroscopic scale the two crystal structures exhibit different engineering properties, such as Young's modulus, electrical resistance, damping behavior, etc. As a consequence, the transition between the two phases gives the possibility to obtain variable and/or tunable properties, i.e. NiTi alloys are able to change and or/adapt their response as a function of external stimuli. In addition, phase transition mechanisms are also associated with high strain recovery capabilities resulting from both thermally-induced and/or stress-induced transformations as described in the following section.

\subsubsection{Thermally-induced martensitic transformation}

When cooling the austenitic structure a thermally-induced martensitic transformation $\left(\mathrm{B} 2 \rightarrow \mathrm{B} 19^{\prime}\right)$ occurs in the temperature range between martensite start temperature $\left(M_{s}\right)$, and martensite finish temperature $\left(M_{f}\right)$. When the martensitic structure is heated the reverse

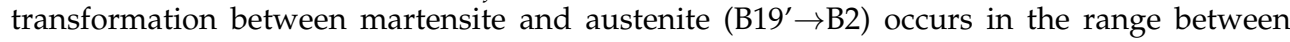


austenite start temperature $\left(A_{s}\right)$ and austenite finish temperature $\left(A_{f}\right)$. These characteristic temperatures, the so called phase transition temperatures (PPTs), can be regarded as material parameters, which depend on the alloy composition and on the thermomechanical processing conditions [1], and can be easily detected by Differential Scanning Calorimetry (DSC) investigation, as schematically shown in Figure 2.

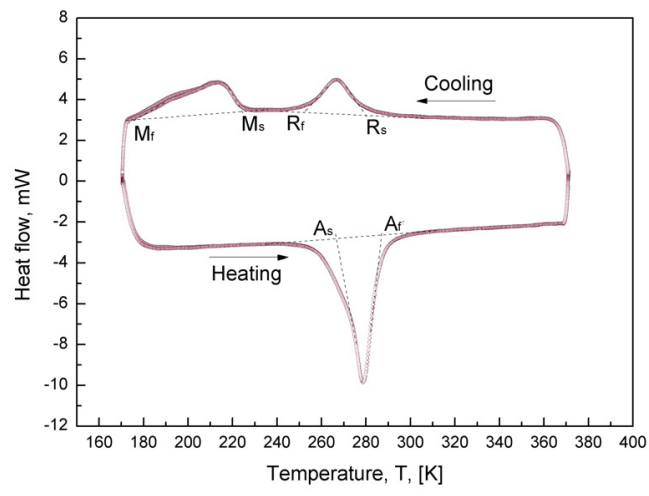

Figure 2. Differential scanning calorimetry thermogram of a NiTi alloy

In addition, transformation from B2 cubic austenite into monoclinic B19' martensite could occur either directly or via an intermediate rhombohedral phase (R-phase), as shown in Figure 2. In particular, the R-phase transformation $(\mathrm{B} 2 \rightarrow \mathrm{R})$ can be observed during cooling from $A_{f}$ to $M_{s}$ prior to martensitic transformation, resulting in a sequential transformation $\mathrm{B} 2 \rightarrow \mathrm{R} \rightarrow \mathrm{B} 19^{\prime}$. However, it is worth noting, that the $\mathrm{B} 2 \rightarrow \mathrm{R}$ transformation is observed only under specific processing conditions of the alloy [33]. In addition, marked differences are normally observed between direct and reverse transformation temperatures as a direct consequence of the thermal hysteretic behavior of the alloy, as illustrated in Figure 3. In particular, this figure shows the thermal hysteresis describing the evolution of the volume fraction of martensite $\left(\xi_{M}\right)$ together with the characteristic transformation temperatures.

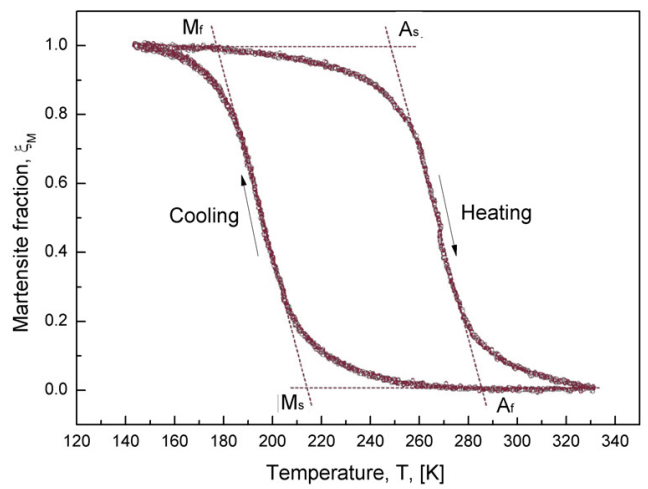

Figure 3. Thermal hysteresis of a NiTi alloy describing the evolution of volume fraction of martensite 


\subsubsection{Stress-induced martensitic transformation}

When a mechanical load is applied to the austenitic structure the stress-induced B2 $\rightarrow$ B19' transformation occurs, corresponding to a plateau in the stress-strain curve of the alloy. If the mechanical load is removed the reverse $\mathrm{B} 19^{\prime} \rightarrow \mathrm{B} 2$ transformation occurs which is related to another stress plateau and allows an almost complete strain recovery. Figure 4 illustrates an example of stress-strain curve of a NiTi alloy exhibiting stress-induced phase transformation mechanisms, together with the characteristic transformation stresses of the alloy, i.e. the stresses for direct $\mathrm{B} 2 \rightarrow \mathrm{B} 19^{\prime}$ transformation $\left(\sigma_{s}^{A M}, \sigma_{f}^{A M}\right)$ and the stresses for reverse $\mathrm{B} 19^{\prime} \rightarrow \mathrm{B} 2$ transformation $\left(\sigma_{s}^{M A}, \sigma_{f}^{M A}\right)$.

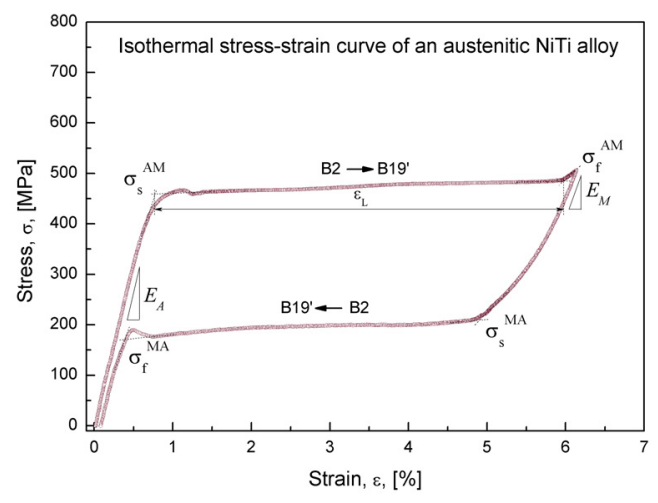

Figure 4. Stress-strain curve of an austenitic NiTi alloy with characteristic transformation stresses.

Figure 4 also illustrates the recovery strain $\varepsilon_{L}$ due to the stress-induced transformation mechanisms, together with the Young's moduli of the two phases $\left(E_{A}\right.$ and $\left.E_{M}\right)$. Another stress-induced microstructural change occurs when a mechanical load is applied to the martensitic structure, i.e. for $T<M_{f}$, the so called detwinning. This mechanism can be regarded as a variant reorientation process and, on the macroscopic scale, it causes large plastic-like deformations which corresponds to a plateau in the stress-strain curve of the alloy. This mechanism is responsible for the shape memory effect as described in the following section. In addition, it is worth noting that NiTi SMAs exhibit a marked temperature dependent stress-strain response, as schematically depicted in Figure 5 . In particular, the temperature dependence of transformation stresses is given by the Clausius-Clapeyron relation of equations 1 :

$$
\frac{d \sigma^{A M}}{d T}=C_{M} ; \frac{d \sigma^{M A}}{d T}=C_{A}
$$

where $C_{M}$ (direct martensitic transformation) and $C_{A}$ (reverse austenitic transformation) are in the range between 5 and $10 \mathrm{MPaK}^{-1}$.

\subsection{Shape memory effect}

Shape Memory Effect (SME) is the ability of a SMA to remember a predetermined shape and to recover this shape even after being subjected to large mechanical deformations (up to $10 \%$ ). In NiTi alloys this property is observed under martensitic conditions, i.e. when $T<M_{f}$, 


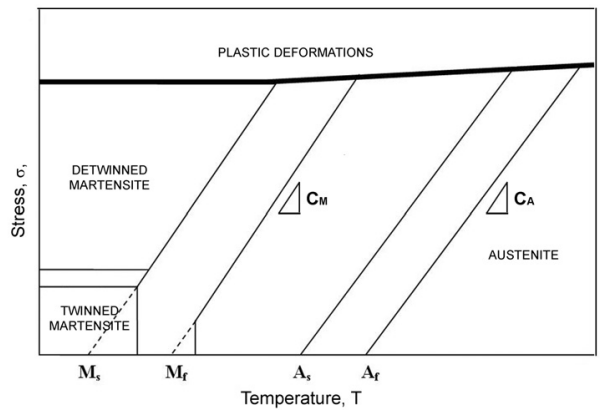

Figure 5. Relation between transformation stresses and temperature according to the Clausius-Clapeyron relation.

and it can be attributed to the combination of two microstructural changes: $i)$ detwinning of martensitic variants and ii) thermally induced phase transformation. Figure 6 shows a schematic depiction of the SME together with the associated phase transition mechanisms (Figure 6.a) and the corresponding stress-strain-temperature response (Figure 6.b).

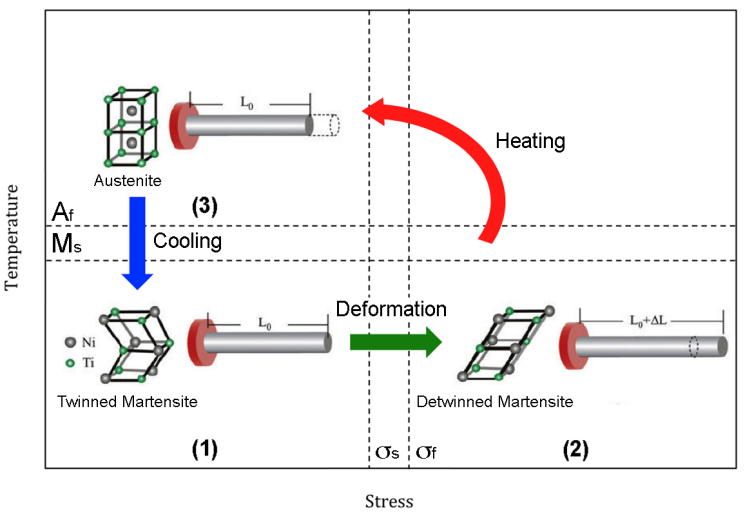

a)

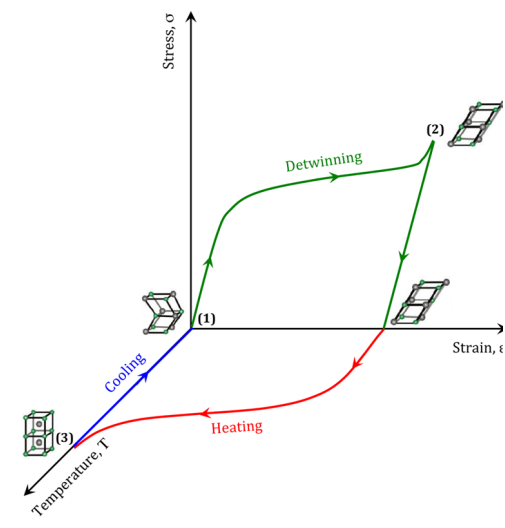

b)

Figure 6. Schematic depiction of the shape memory effect: a) phase transition mechanisms and b) stress-strain-temperature response

In particular, Figs. 6 show that if a mechanical load is applied to the twinned martensitic structure (1), i.e. for $T<M_{f}$, detwinning occurs at a given critical stress value which corresponds to large plastic-like deformations (up to 10\%) through a plateau in the stress-strain curve (2). In fact, these deformations persist after complete unloading as only elastic recovery of the detwinned structure is observed. However, if the material is heated up to the austenite finish temperature $\left(T>A_{f}\right)$ a complete thermally induced phase transformation occurs from the detwinned martensitic structure to the austenitic one (3) and, on the macroscopic scale, this transformation allows a complete shape recovery. Finally, if the material is cooled down to the martensite finish temperature $\left(T<M_{f}\right)$ it is able to remember 
its original twinned martensitic structure (1). This unusual functional property is also known as one-way shape memory effect (OWSME) as it defines the ability of material to remember just one shape, the cold one $\left(T<M_{f}\right)$, and to recover this shape after being mechanically deformed. However, under specific thermo-mechanical treatments NiTi alloys could exhibit another shape memory mechanism, the so called two-way shape memory effect (TWSME), i.e. they are able to remember a cold shape, linked to the martensitic structure, and a hot shape, linked to the austenite. As a consequence, during repeated heating and cooling, the material changes its shape in a reversible way, through a hysteresis loop, as schematically illustrated in Figure 7.
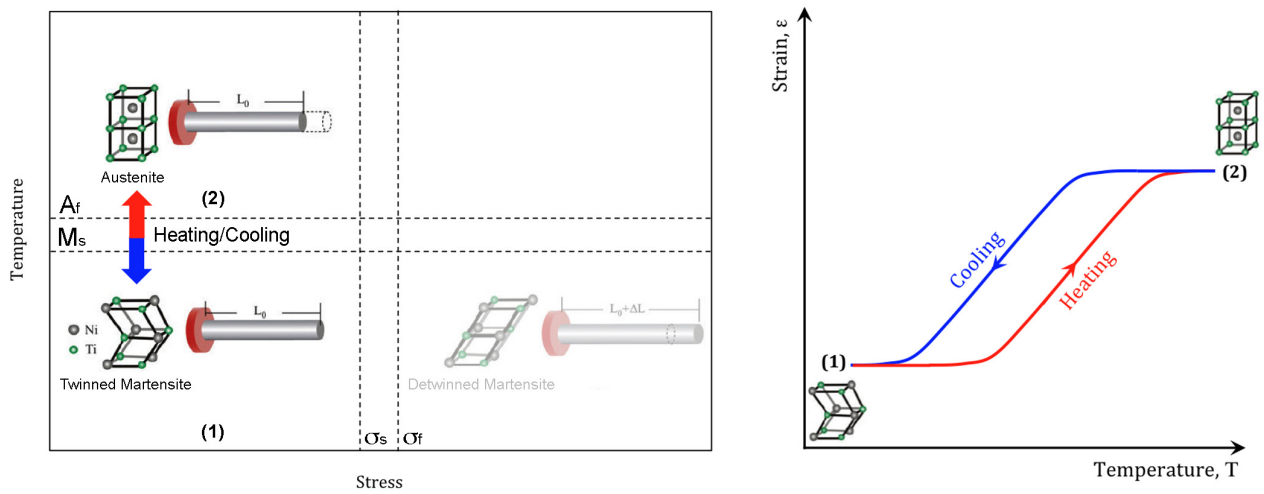

Figure 7. Schematic depiction of the two-way shape memory effect: a) phase transition mechanisms and b) strain-temperature hysteretic response

In particular, TWSME can be induced by proper thermomechanical procedures, the so-called training, which usually involve repeated deformations and transformations between austenite and martensite. This thermomechanical process produces a dislocation structure and, consequently, creates an anisotropic stress field that benefits the formation of preferentially oriented martensite variants [32], resulting in a macroscopic shape change between the phase transition temperatures.

Figure 8 shows an example of the thermomechanical cycle, which is composed of four subsequent steps: 1) strain controlled uniaxial loading up to a training deformation $\varepsilon_{t r}, 2$ ) complete unloading at the same rate and recording of the residual strain $\varepsilon_{r}, 3$ ) heating up to the austenite finish temperature $A_{f}$, in stress free conditions, to activate SME and measuring the recovery deformation $\varepsilon_{r e}$ and permanent strain $\varepsilon_{p}$, and 4) cooling down to the martensite finish temperature $M_{f}$, in stress free conditions, and recording the induced two-way shape memory strain $\varepsilon_{t w}$. Experimental measurements have been carried out in [28] where several training cycles have been executed with a training deformation $\varepsilon_{t r}=5.5 \%$. Each training cycle starts from the end of the cooling stage of the previous one, so that the total deformation at the $i-t h$ cycle, $\varepsilon_{\text {tot }(i)}$, can be defined as follows:

$$
\varepsilon_{t o t(i)}=\varepsilon_{t r(i)}+\varepsilon_{p(i-1)}+\varepsilon_{t w(i-1)}
$$

Figure 9 reports the measured $\varepsilon_{t w}, \varepsilon_{p}, \varepsilon_{p e}$, and $\varepsilon_{t o t} v s$ the number of training cycles. The figure clearly shows that the two-way shape memory strain $\varepsilon_{t w}$ increases with increasing the number 


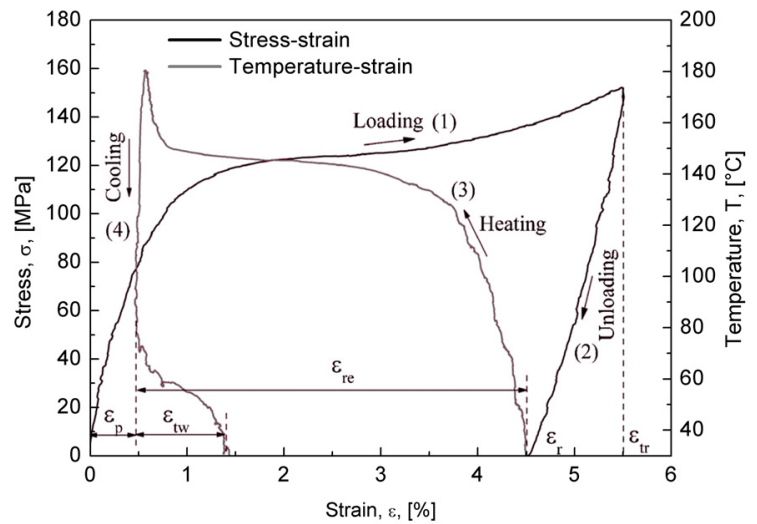

Figure 8. Example of training cycle: 1) loading, 2) unloading, 3) heating up to $A_{f}$, and 4) cooling down to $M_{f}$ [28].

of training cycles, and a similar behavior is observed for the permanent deformation $\varepsilon_{p}$, the strain recovery $\varepsilon_{r e}$, and the total deformation $\varepsilon_{t o t}$. In particular, $\varepsilon_{t w}$ increases from $1.0 \%$ at the first training cycle to $2.8 \%$ after six cycles; $\varepsilon_{p}$ and $\varepsilon_{r e}$ increase from $1.5 \%$ to $4.2 \%$ and from $4 \%$ to $6 \%$, respectively, and, consequently, $\varepsilon_{\text {tot }}$ increases from $5.5 \%$ to $11.8 \%$. In Figure 10 , the

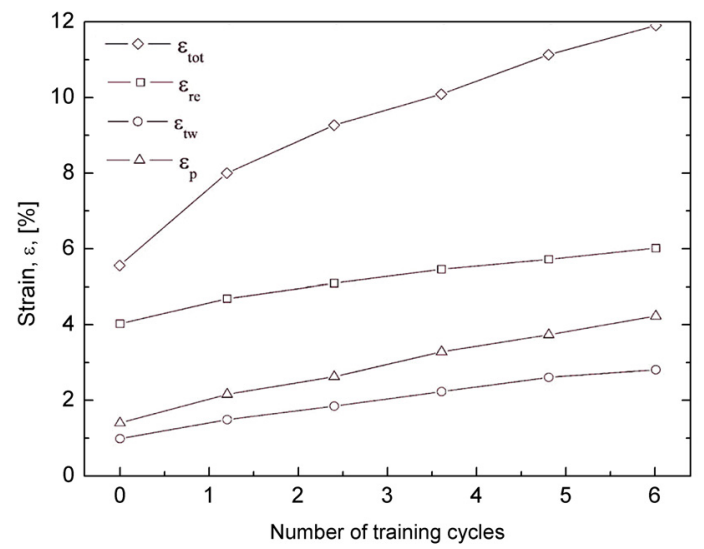

Figure 9. Measured deformations versus number of training cycles: $\varepsilon_{t w}, \varepsilon_{p}, \varepsilon_{p e}$, and $\varepsilon_{t o t}$ [28].

measured thermal hysteresis behavior strain $v s$ temperature, describing the TWSME of the trained material, is shown. In particular, Figure 10.a illustrates the stress-free hysteresis loop, together with the PTTs, while in Figure 10.b the stress-free thermal hysteresis loop is compared with those obtained under a tensile stress of $50 \mathrm{MPa}$ and $100 \mathrm{MPa}$. The comparison clearly shows and a systematic increase in $\varepsilon_{t w}$, as well as in all PTTs, when increasing the applied stress $\sigma$. In particular, the increase of $\varepsilon_{t w}$ is attributed to $i$ ) the variation of Young's modulus in the thermal hysteresis behavior between martensite and austenite and ii) the increased volume fraction of favorably oriented martensite variants with increasing external stress. 


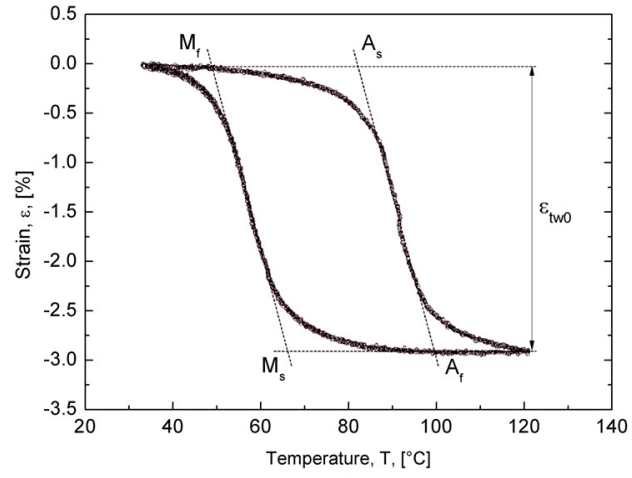

a)

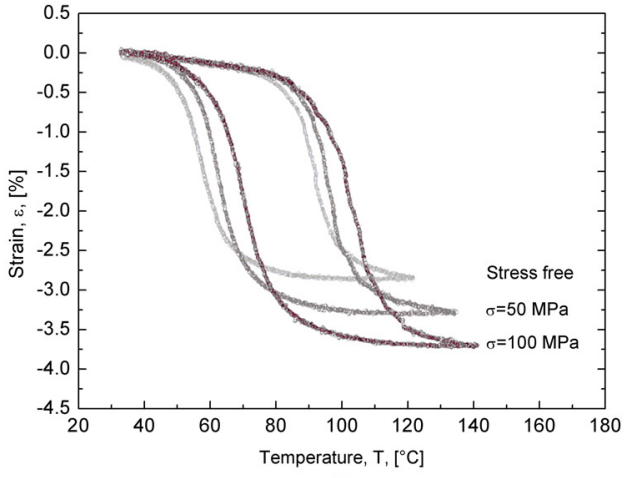

b)

Figure 10. Thermal hysteresis behavior of the trained material strain $v$ s temperature: a) stress-free hysteresis loop with an highlight of the PTTs and b) effects of the applied tensile stress [28].

\subsection{Pseudoelastic effect}

The pseudoelastic (PE) effect in NiTi alloys consists in the high strain recovery capability (up to $10 \%$ ) observed during isothermal loading/unloading cycles carried out at temperature $T>A_{f}$, i.e. when the alloy is in austenitic conditions. This functional property can be directly attributed to the reversible stress-induced martensitic transformations as discussed in section 2.1.2. In particular, Figure 11 illustrates that if a mechanical load is applied to austenitic structure (1) the B2 $\rightarrow$ B19' transformation occurs and, on the macroscopic scale, large mechanical deformation are achieved through a stress-strain transformation plateau (2). However, if the mechanical load is removed the reverse B19' $\rightarrow \mathrm{B} 2$ transformation occurs and, consequently, the material is able to recover its original shape through an unloading plateau in the stress strain curve. However, the reverse transformation occurs at lower stress values resulting in a marked stress-strain hysteretic behavior.

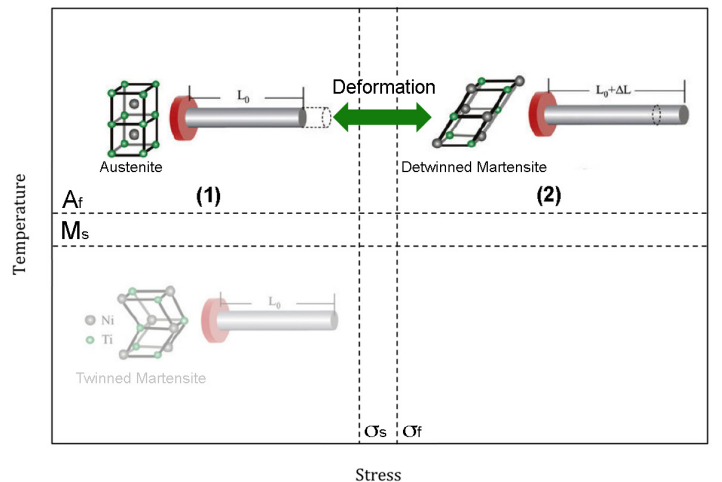

a)

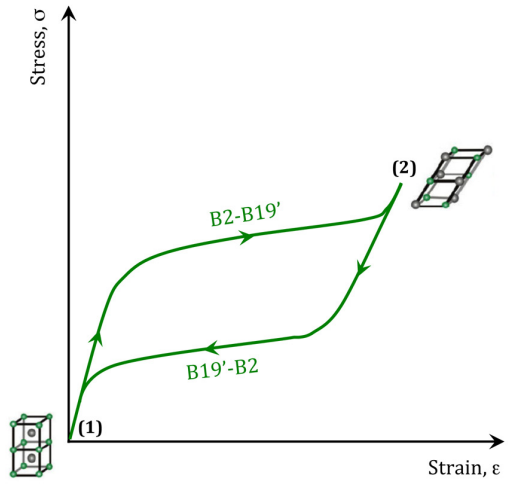

b)

Figure 11. Schematic depiction of the pseudo elastic effect: a) stress-induced phase transition mechanisms and b) stress-strain hysteretic response 
It is worth noting that Figure 11.b illustrates the nominal stress-strain behavior of a pseudoelastic SMA, while NiTi alloys exhibit a marked evolution of the stress-strain hysteretic behavior in the first mechanical cycles, up to a stable response, due to the formation of stabilized martensite [32], which causes a reduction of the pseudoelastic recovery of the SMA. In particular, Figure 12 illustrates the evolution of the stress-strain hysteretic behavior of the material in the first 25 mechanical cycles for a fixed value of total strain $\varepsilon_{t o t}=3.5 \%$. These data were obtained from experimental testing of a commercial pseudoelastic NiTi alloy [30]; the figure clearly shows a marked reduction of the pseudoelastic recovery, from $3.5 \%$ to about $3 \%$, but the stress-strain loops becomes stable after 20 cycles. Furthermore, as reported in section

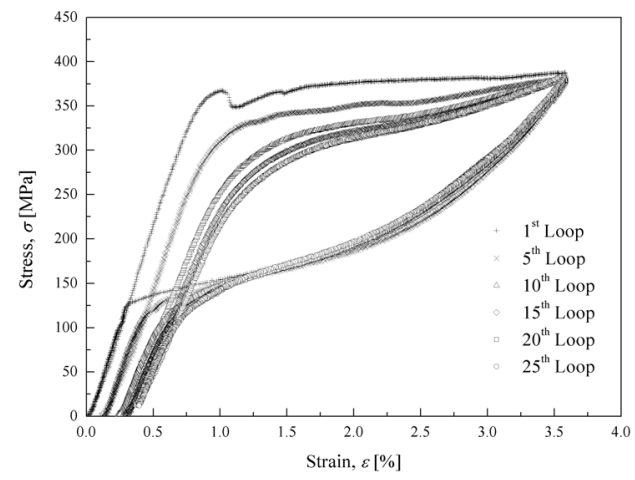

Figure 12. Evolution of the stress-strain response of a commercial pseudoelastic NiTi alloy during the first 25 mechanical cycles for a maximum applied deformation $\varepsilon_{\text {tot }}=3.5 \%$ [30]

2.1.2, the critical stresses for direct and inverse phase transformation are significantly affected by the temperature, according to the Clausius-Clapeyron constant (Equation 1), as illustrated in the experimentally measured curves in Figure 13 [30]. These curves have been obtained from isothermal loading unloading cycles, carried out at increasing values of the testing temperature for $T>A_{f}(303 \mathrm{~K}-328 \mathrm{~K})$, by using a SMA with a stable pseudoelastic response. The analysis of the data in Figure 13 allows to obtain the value of the Clausius-Clapeyron constant $\left(C_{M}=C_{A}=8.7 \mathrm{MPaK}^{-1}\right)$.

\section{Hysteresis modeling: basics}

A one-dimensional numerical approach to simulate the stress-strain and strain-temperature hysteresis behavior of SMAs is described in this section. In particular, in a purely phenomenological way, the hysteresis loop is modeled by a Prandtl-Ishlinskii hysteresis operator $H$ [26]; the basic idea of this approach consists in modeling the non-linear hysteretic behavior by a weighted superposition of many elementary hysteresis operators, such as the backlash operators $\mathrm{Hr}$, as schematically illustrated in Figure 14.

$$
H=\{w\}^{T}\left\{H_{r}\right\}
$$

where $\left\{H_{r}\right\}$ is the vector of backlash operators and $\{w\}$ is the corresponding vector of weights. As shown in Figure 14.a, each backlash operator $H_{r i}$ is characterized by its dead band width $d w_{i}$, while the corresponding weight $w_{i}$ represents the slope of the oblique lines of the operator. As illustrated in Figure 14.b, which represent a generic $x(t)-y(t)$ hysteretic 


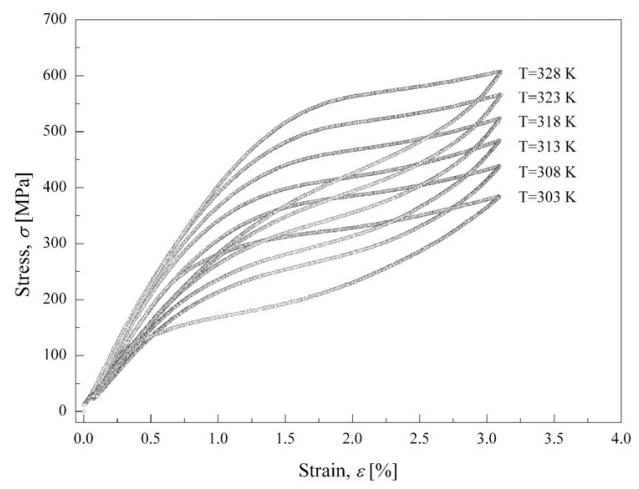

Figure 13. Isothermal stress-strain hysteresis loops of a commercial pseudoelastic NiTi alloy as a function of the testing temperature [30].

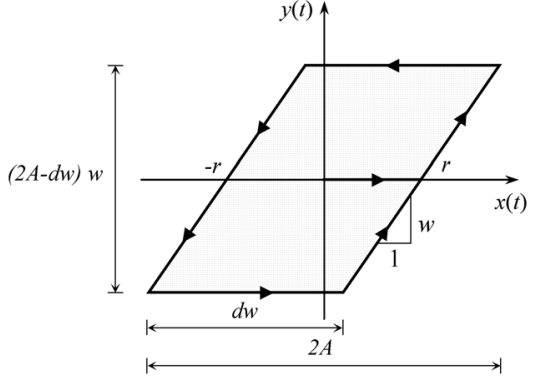

a)

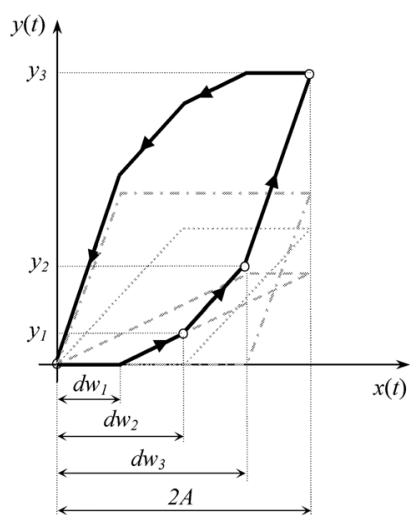

b)

Figure 14. Schematic depiction of the hysteresis operators: a) elementary backlash operator $H_{r}$ and b) Prandtl-Ishlinskii hysteresis operator $H$ given by weighted superposition of elementary hysteresis operators.

behavior, the proposed approach consists of modeling the hysteretic loop by a linear piecewise discretization. The accuracy of the model can be improved by increasing the total number of linear pieces, which represent the total number of the backlash operators. The problem of modeling the hysteretic behavior, starting from the experimental measurements, is now reduced to the determination of the deadband width vector $\{d w\}$ of the backlash operators and the associated gain vector $\{w\}$. In particular, the parameters of the model can be easily identified by the outer loop of the hysteretic region by using the following simple relation:

$$
y_{k}=\sum_{i=1}^{k}\left(d w_{k+1}-d w_{i}\right) w_{i}
$$

where $y_{k}$ is the output value of the lower branch of the loop in the generic point of discontinuity $k$, as shown in Figure 14.b. The vector $\{d w\}$ is a user defined discretization of 
the total amplitude of the input signal. Equation 4 can be rewritten in matrix form as follows:

$$
\{y\}=[A]\{w\}
$$

where the matrix $[A]$ is constructed, for a given $\{d w\}$ vector, by using equation 4; the unknown vector $\{w\}$ can be found by solving a system of $N$ linear equations, where $N$ is the total number of backlash operators, as follows:

$$
\{w\}=[A]^{-1}\{y\}
$$

The main drawback of the Prandtl-Ishlinskii approach consists in the fact that only loops with an odd symmetry to the relative center can be modeled; in fact the symmetry of the elementary hysteresis operator, with respect to the center of the loop, persists under linear superposition. However, this drawback can be overcome by using a modified Prandtl-Ishlinksii operator, as described in details in [26], in which a weighted superposition of saturation operators is combined with the hysteresis operator. The parameters of this sub model, such as saturation limits $\{S\}$ and associated gains $\left\{w_{s}\right\}$, can be identified by using a procedure similar to that described above.

\subsection{Modeling of pseudoelastic effect}

The numerical model described in this section is able to simulate the pseudoelastic effect of a shape memory alloy [30], i.e. the the stress-strain $(\sigma-\varepsilon)$ hysteretic behavior, based on the Prandtl-Ishlinksii operator and on the assumptions reported in the following.

\subsubsection{Basic assumptions}

Figure 15 shows the stabilized stress-strain hysteretic behavior of a commercial NiTi alloy, i.e. the response of the material after the first training cycles (see Figure 12), for different values of the applied deformation. The figure also illustrates the Young's moduli of austenite and detwinned martensitic structures, $E_{A}$ and $E_{M}$, together with the generic young's modulus of the alloy, $E\left(\xi_{M}\right)$, corresponding to an incomplete stress induced martensitic transformation, i.e. as a function of the martensite fraction $\xi_{M}\left(0<\xi_{M}<1\right)$. In particular, $E_{A}$ represents the slope of the early stage of the stress-strain loading curve, $E_{M}$ is measured from the unloading curve of a complete martensitic transformation $\left(\xi_{M}=1\right)$, while $E\left(\xi_{M}\right)$ is obtained from the unloading path of an incomplete phase transformation.

The total strain $\varepsilon$ can be decomposed in elastic and a transformation strain components, $\varepsilon_{e l}$ and $\varepsilon_{t r}$, respectively:

$$
\varepsilon=\varepsilon_{e l}+\varepsilon_{t r}
$$

where the elastic strain can be expressed as a function of the applied stress, $\sigma$, and of the Young's modulus, $E\left(\xi_{M}\right)$, of the material:

$$
\varepsilon_{e l}=\frac{\sigma}{E\left(\xi_{M}\right)}
$$

As schematically shown in Figure 15 the Young's modulus changes during stress-induced phase transformation between austenite and martensite, i.e. it decreases from $E_{A}$ to $E_{M}$, and 


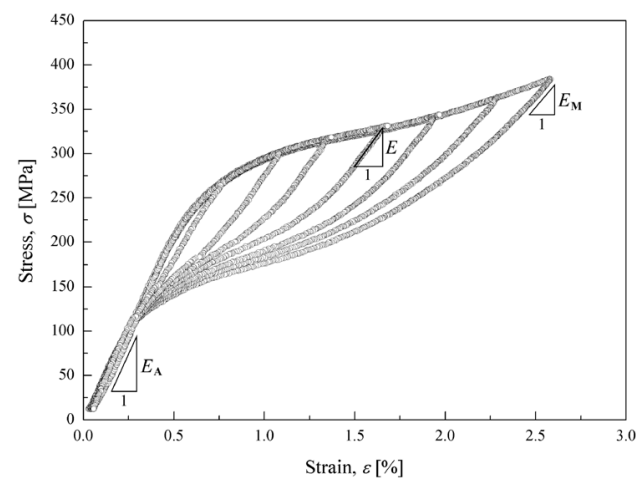

Figure 15. Stress-strain hysteretic behavior of a commercial NiTi alloy together with the Young's moduli of austenite and detwinned martensite [30].

it is assumed to be dependent on the volume fraction of martensite $\xi_{M}$ according to the Reuss formula [20]:

$$
\frac{1}{E\left(\xi_{M}\right)}=\frac{\xi_{M}}{E_{M}}+\frac{1-\xi_{M}}{E_{A}}
$$

The evolution of martensite is assumed to be a linear function of the stress in the stress-strain transformation curves, i.e. in the range $\left(\sigma_{s}^{A M}, \sigma_{f}^{A M}\right)$ in the loading stage and $\left(\sigma_{s}^{M A}, \sigma_{f}^{M A}\right)$ in the unloading stage. In particular, the evolution rule for a complete transformation, can be expressed as follows:

$$
\xi_{M}= \begin{cases}\frac{\sigma-\sigma_{s}^{A M}}{\sigma_{f}^{A M}-\sigma_{s}^{A M}} & \text { Loading path } \\ 1-\frac{\sigma-\sigma_{s}^{M A}}{\sigma_{f}^{M A}-\sigma_{s}^{M A}} & \text { Unloading path }\end{cases}
$$

The assumptions given by equations (9) and (10) have been validated by experimental measurements of the Young's modulus, as reported in Figure16. In particular, the figure shows the measured values of the Young's modulus as a function of the applied stress, on the direct stress-strain transformation plateau, together with the predictions of the Reuss formula, and a satisfactory agreement is observed.

However, it's worth noting that the evolution of martensite is characterized by a hysteretic behavior, i.e. it is stress path dependent, and equations (9) and (10) do not correctly predict the effects of incomplete transformations. These effects are simulated mathematically, by using a backlash operator, as schematically illustrated in Figure 17; in particular, Figure 17.a shows the stress vs time path, while Figure 17.b illustrates the evolution of $\xi_{M}$ and $1 / E\left(\xi_{M}\right)$ vs the applied stress. Furthermore, the continuous lines in Figure 17.b are relative to a complete martensitic transformation, i.e. $\xi_{M}$ increases from 0 to 1 , while the dashed lines show the effects of an incomplete transformation. Due to the modification in Young's modulus during the phase transformation between austenite and martensite, as shown in Figure 17.b, both elastic and transformation strain components are represented by a hysteretic behavior and it can be calculated using equations (7-10); as an example in Figure 18 a typical $\sigma-\varepsilon$ hysteresis loop for a complete phase transformation is compared with the corresponding computed elastic strain $\left(\sigma-\varepsilon_{e l}\right)$ and transformation strain $\left(\sigma-\varepsilon_{t r}\right)$ hysteresis loops. 


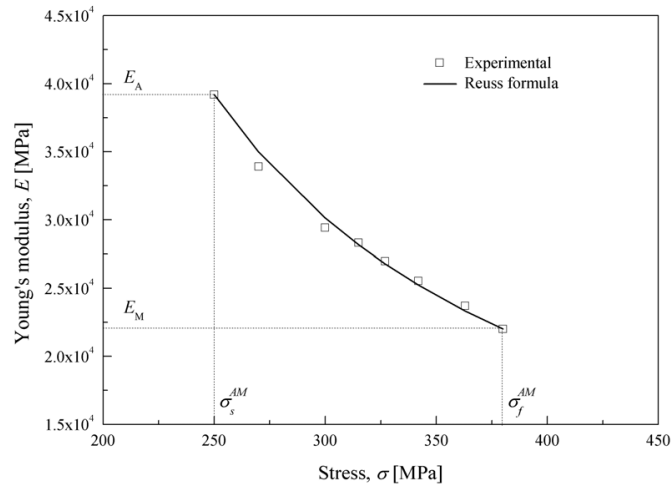

Figure 16. Evolution of the Young's modulus as a function of the applied stress: experimental measurements vs simulations [30].

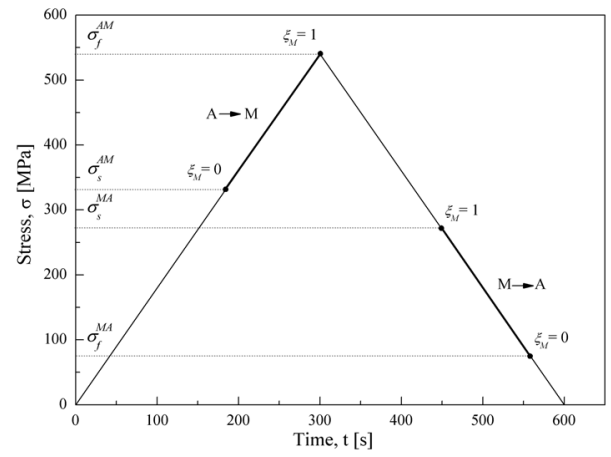

a)

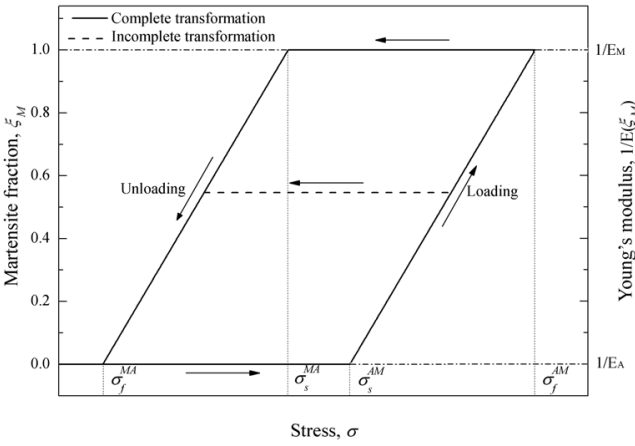

b)

Figure 17. Evolution rule of the martensite fraction $\xi_{M}$ in the tension cycle: a) example of stress-time path and b) simple hysteresis model to predict the Young's modulus $E\left(\xi_{M}\right)$ [30].

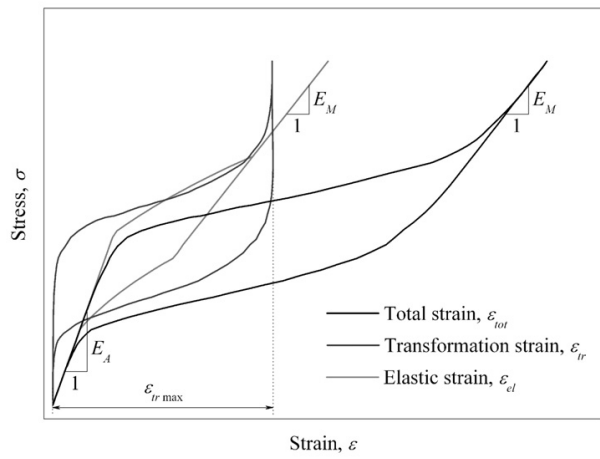

Figure 18. Stress strain $(\sigma-\varepsilon)$ hysteresis loops: elastic strain $\varepsilon_{e l}$ and transformation strain $\varepsilon_{t r}$ [30]. 


\subsubsection{Numerical flowchart}

Based on the Prandtl-Ishlinskii hysteresis modeling approach together with the model assumptions described in the previous section, a one-dimensional numerical model can be easily developed by using commercial software packages. In particular, Figure 19 illustrates an implementation of the model in the MatlabSimulink ${ }^{T M}$ platform, in which the two strain components, $\varepsilon_{e l}$ and $\varepsilon_{t r}$, are treated separately. As clearly illustrated in Figure 19, both stress

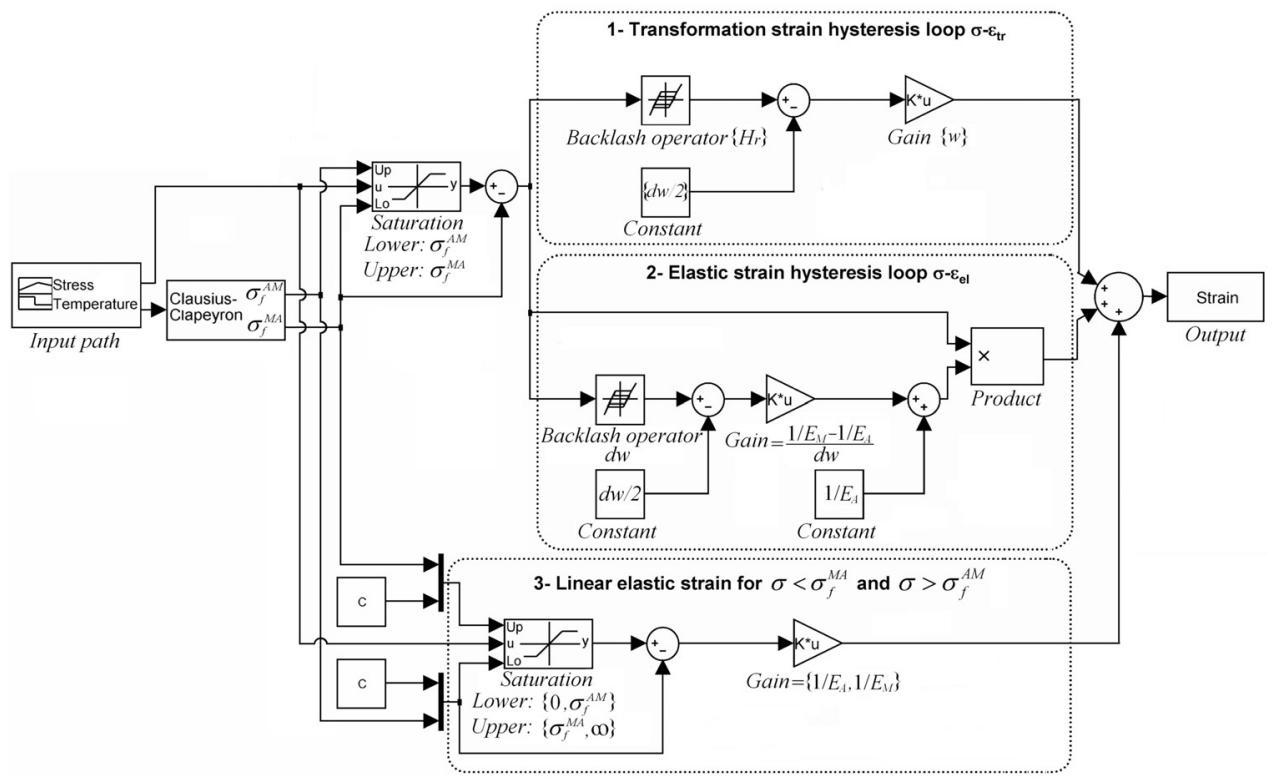

Figure 19. Flowchart of a MatlabSimulink ${ }^{T M}$ model to simulate the pseudoelastic effect in SMAs [30].

and temperature are taken as input variables, and the critical stresses are calculated, based on the current value of the temperature, by using the Clausius-Clapeyron relation (equation 1). In the model the saturation operators are used to identify the stress range where the material exhibits the hysteretic behavior $\left(\sigma_{f}^{M A}<\sigma<\sigma_{f}^{A M}\right)$, and the dead zones of transformation where only elastic deformation of austenite $\left(\sigma<\sigma_{f}^{M A}\right)$ or martensite $\left(\sigma>\sigma_{f}^{A M}\right)$ occurs. Three different sub-models are highlighted in Figure 19 which simulate the $\sigma-\varepsilon_{t r}$ and $\sigma-\varepsilon_{e l}$ hysteresis loops, and the linear elastic response of the material in austenitic and martensitic conditions. In particular, a Prandtl-Ishlinskii hysteresis operator, was used to model the $\sigma-\varepsilon_{t r}$ hysteretic behavior, a single backlash operator was adopted to model the $\sigma-\varepsilon_{e l}$ loop (see Figure 17.b), and a gain operator was used to describe the linear elastic response for $\sigma<\sigma_{f}^{M A}$ and for $\sigma>\sigma_{f}^{A M}$. The parameters of the Prandtl-Ishlinskii operator describing the $\sigma-\varepsilon_{t r}$ hysteretic behavior, i.e. the deadband width vector $\{d w\}$ and the associated gain vector $\{w\}$ are determined from an experimentally measured stress-strain hysteresis loop by using the procedure described in section 3. In particular, as shown in Figure 20, the generic input and output variables $(x$ and $y)$ can be regarded as the stress and strain values $(\sigma$ and $\varepsilon)$, respectively. As a consequence, the vector $\{d w\}$ represents a user defined discretization of 
the stress amplitude while the vector $\{w\}$ can be determined by using equation (6), where the vector $\{y\}$ is the vector of strain values $\{\varepsilon\}$. The computational procedure to obtain the aforementioned model parameters from an experimentally measured hysteretic loop can be easily implemented, which generates the vector $\{d w\}$, by a partition of the input stress amplitude, and calculates the unknown vector of weight $\{w\}$ by solving a system of $N$ linear equations, where $N$ is the number of backlash operators.

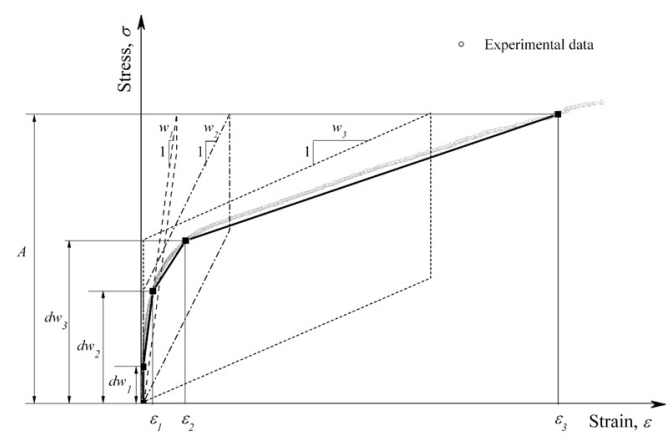

Figure 20. Loading branch of the stress-strain hysteresis loop and linear piecewise discretization obtained by a weighted superposition of three backlash operators [30].

\subsubsection{Numerical results vs experiments}

The accuracy of the numerical method is illustrated by comparisons with experimentally measured hysteresis loops, by using he thermo-mechanical parameters reported in [30] $\left(E_{A}=39 \mathrm{GPa}, E_{M}=20 \mathrm{GPa}, \sigma_{s}^{A M}=260 \mathrm{MPa}, \sigma_{f}^{A M}=385 \mathrm{MPa}, \sigma_{s}^{M A}=250 \mathrm{MPa}, \sigma_{f}^{M A}=125 \mathrm{MPa}\right.$, $\left.C_{A}=C M=10.3 \mathrm{MPa} / \mathrm{K}\right)$. Figure 21 shows the stress-strain hysteretic behavior of the SMA for a stress path which involves several incomplete stress-induced martensitic transformations $(A \rightarrow M)$, by repeated isothermal tension cycles $(T=303 \mathrm{~K})$ carried out between $\sigma_{\min }=0$ and decreasing values of $\sigma_{\max }<\sigma_{f}^{A M}$. In particular, the comparison between numerical simulations and experimental measurements, illustrated in Figure 21.a, clearly shows a satisfactory accuracy of the model in predicting the non-linear stress-strain hysteretic behavior of the material; the figure also shows that the model is able to capture the modification of Young's modulus in the stress-strain transformation curve, as it correctly predicts the change in the slopes of the unloading curves. Furthermore, Figure 21.b shows the evolutions of the transformation strain, $\varepsilon_{t r}$, and elastic strain, $\varepsilon_{e l}$, for the same input stress path of Figure 21.a. As illustrated in section 3.1.1, both $\varepsilon_{t r}$ and $\varepsilon_{e l}$ are characterized by hysteretic behaviors, which are due to the mismatch between the critical stresses in the stress induced transformations and the modification of Young's modulus in the stress-strain transformation curve, respectively.

Figures 22 show comparisons between numerical predictions and experimental measurements for two input isothermal stress paths $(T=303 \mathrm{~K})$ which involve incomplete $M \rightarrow A$ transformations (Figure 22.a) and both incomplete $A \rightarrow M$ and $M \rightarrow A$ transformations (Figure 22.b). In particular, Figure 22.a shows the hysteretic response of the material for a stress path which is composed of some subsequent tension cycles between increasing values of $\sigma_{\min }<\sigma_{f}^{M A}$ and $\sigma_{\max }=$ const, while Figure 22.b is relative to a stress 


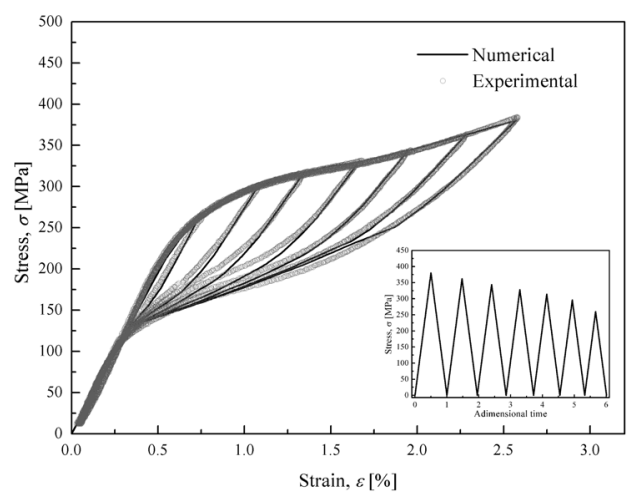

a)

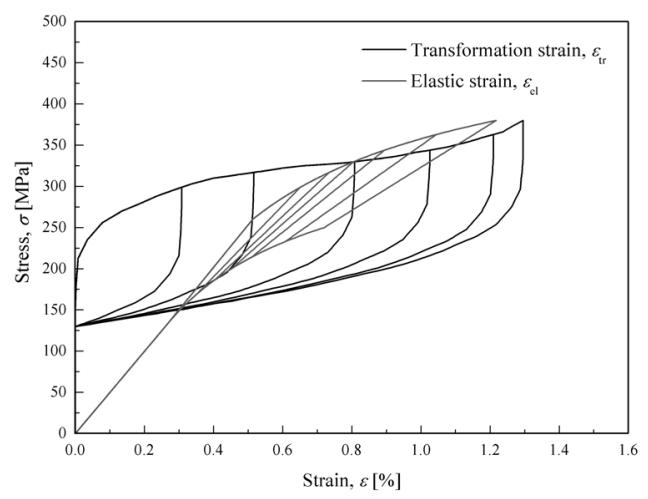

b)

Figure 21. Numerical simulation for isothermal stress cycles with incomplete $A \rightarrow M$ transformations: a) comparison with experimentally measured loops and b) evolution of transformation strain, $\varepsilon_{t r}$, and elastic strain, $\varepsilon_{e l}[30]$.

path which involves different subsequent tension cycles carried out between increasing values of $\sigma_{\min }>\sigma_{f}^{M A}$ and decreasing values of $\sigma_{\max }<\sigma_{f}^{A M}$; both figures shows good agreements between experiments and numerical simulations.

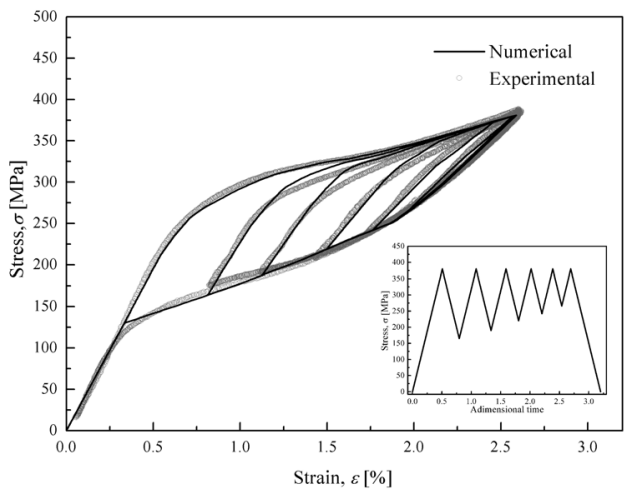

a)

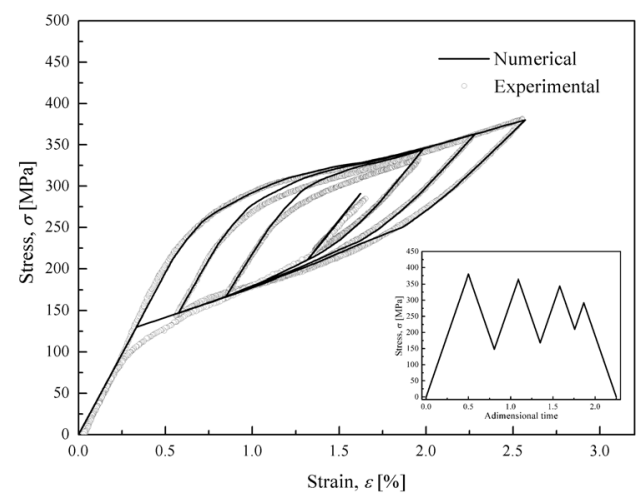

b)

Figure 22. Comparison between numerical simulations and experimentally measured hysteresis loops for isothermal stress cycles with: a) incomplete $M \rightarrow A$ transformations and b) incomplete $A \rightarrow M$ and $M \rightarrow A$ transformations [30].

\subsection{Modeling of two-way shape memory effect}

The numerical model described in this section is able to simulate the two-way shape memory effect of a trained NiTi based shape memory alloy, i.e. the the strain-temperature $(\varepsilon-T)$ hysteretic behavior [27-29]. Furthermore, the model is able to capture the effects of applied stress on the thermal recovery of the alloy. 


\subsubsection{Basic assumptions}

In a pure phenomenological way, the variation of the two-way shape memory strain, $\varepsilon_{t w}$, with increasing the applied stress (see Figure 10.b) can be attributed to two different mechanisms: i) the variation of Young's modulus in the thermal hysteresis behavior between martensite and austenite, $i$ ) the increased volume fraction of favorably oriented martensite variants with increasing external stress.

$$
\Delta \varepsilon_{t w}=\varepsilon_{t w}-\varepsilon_{t w 0}=\left(\frac{1}{E_{M}}-\frac{1}{E_{A}}\right) \sigma+c \sigma
$$

where subscript 0 indicate the stress-free condition, $E_{M}$ and $E_{A}$ represent the Young's moduli of martensite and austenite, respectively, while $c$ can be assumed as a material constant. In particular, the first term at the right end side of equation 11, indicated as $\Delta \varepsilon_{\text {mech }}$, describe the first effect while the last terms, namely $\Delta \varepsilon_{m e m}$, take into account the second effect. Young's moduli $E_{M}$ and $E_{A}$ can be measured by isothermal tensile tests carried out at temperatures $T<M_{f}$ and $T>A_{f}$, respectively, while the parameter $c$ can be obtained from experimental measurements of the two-way shape memory strain carried out at different value of the applied stress. In the following the values of the parameters reported in [28] have been used $\left(E_{M}=36 * 10^{3} \mathrm{MPa}, E_{A}=67 * 10^{3} \mathrm{MPa}, \mathrm{c}=8.5 * 10^{-5} \mathrm{MPa} a^{-1}\right)$. Two simple mathematical functions, obtained by a numerical fitting of the experimental data, are used to describe the phase transformation kinetics. In particular, as reported in the model by Tanaka and Nagaki [13], the heating and cooling branches of the hysteresis loop can be represented by two exponential curves:

$$
\varepsilon= \begin{cases}\left.\varepsilon_{t w} e^{a_{A}\left(A_{f}-T+\delta T_{M}\right.}\right) & \mathrm{M} \rightarrow \mathrm{A} \\ \varepsilon_{t w}\left[1-e^{a_{M}\left(M_{f}-T+\delta T_{A}\right.}\right) & \mathrm{A} \rightarrow \mathrm{M}\end{cases}
$$

where $\varepsilon_{t w}, M_{S}$, and $A_{s}$ are functions of the applied stress, as reported in Equations 11 and $1 ; a_{M}, a_{A}, \delta T_{M}$, and $\delta T_{A}$, which define the shape of the heating and cooling branches of the loop, can be identified by a numerical fitting of the experimental data. Figure 23.a shows a comparison between experimental measurement and exponential curves; in the figure, points $P_{1}$ and $P_{2}$ represent the range where the numerical fitting is executed to identify the parameters of the heating branch of the loop, while points $P_{3}$ and $P_{4}$ are relative to the cooling branch. In Figure 23.b, a linear fitting between points $P_{1}$ and $P_{2}$ of the experimental data in the $T-\log \varepsilon$ plane is shown, where the slope of the line defines the parameter $a_{A}$ and the intersection with the $\log \varepsilon$ axis allows us to obtain the parameter $\delta T_{M}$. If the loop is characterized by an odd symmetry with respect to its center, as is quite well observed in the investigated material, the same values can be assumed for the constants $a_{A}$ and $a_{M}$ and $\delta T_{A}$, and $\delta T_{M}$. The two exponential curves describe the two branches of the hysteresis loop in a parametric way for a generic value of the applied stress by using Equations 1 and 11. Starting from the curves $\varepsilon-T$, the numerical method based on the Prandtl-Ishlinksii operator can be developed, which is able to predict the output response for a generic temperature path, as decribed in the following section.

\subsubsection{Numerical flowchart}

The numerical method described above can be easily implemented in a MatlabSimulink ${ }^{T M}$ model, as shown in Figure 24, by a modified Prandtl-Hishlinkii hysteresis operator. The 


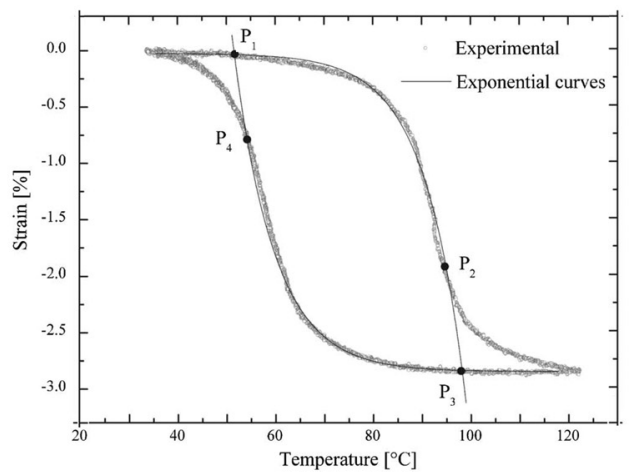

a)

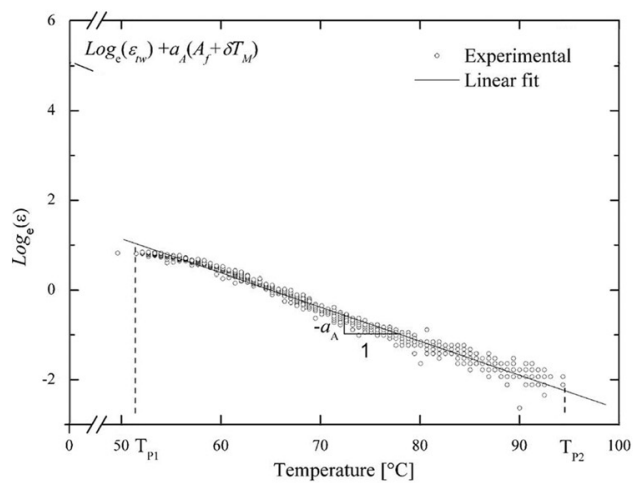

b)

Figure 23. Numerical fitting of the experimental data: a) comparison between exponential curves and experimental measurements; b) numerical fitting in the $T-\log \varepsilon$ plane to identify the parameters of the exponential curve in the heating branch of the hysteresis loop [28]

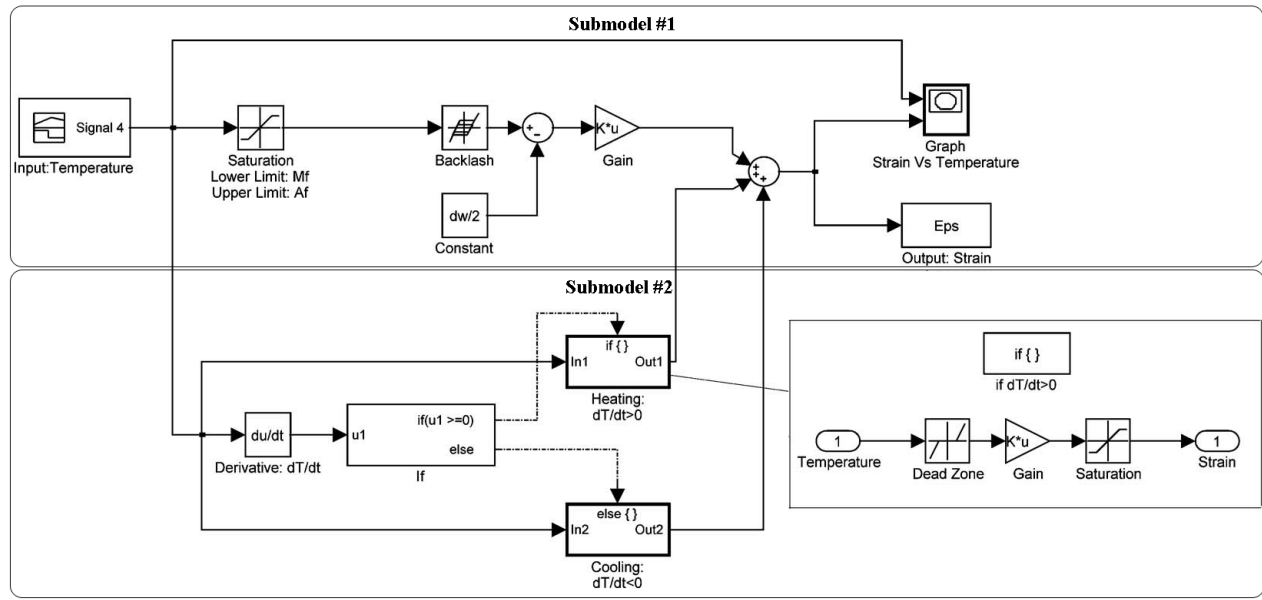

Figure 24. Flowchart of a MatlabSimulink ${ }^{T M}$ model to simulate the two way shape memory effect in SMAs.

Prandtl-Hishlinkii operator is implemented in the submodel \#1 of Figure 24, by a weighted superposition of several backlash operators, and the corresponding parameters, i.e. the deadband width vector $\{d w\}$ and the associated gain vector $\{w\}$, are determined from the exponential curves of equation 12, which in turn are obtained from a fitting of experimental data $(T-\varepsilon)$ as illustrated in Figure 23. In particular, the generic input and output variables $(x$ and $y)$ can be regarded as the temperature and strain values ( $T$ and $\varepsilon$ ), respectively. As a consequence, the vector $\{d w\}$ represents a user defined discretization of the temperature amplitude while the vector $\{w\}$ can be determined by using equation (6), where the vector $\{y\}$ is the vector of strain values $\{\varepsilon\}$ obtained from equation $12\left(\varepsilon_{i}=\varepsilon\left(T_{i}\right)\right)$. The saturation operator in the submodel $\# 1$ is used to simulate the dead zones of transformation, i.e. the 
material behavior when the temperature is above $A_{f}$ during heating and below $M_{f}$ in the cooling branch of the hysteresis loop. In particular, this operator imposes upper and lower bounds on the temperature, which are $A_{f}$ and $M_{f}$, respectively, so that when the temperature is outside these bounds the signal is clipped to the upper or lower bounds. In Figure 25.a, the response of the submodel \#1, in terms of strain-temperature loop, is illustrated for a thermal cycle between the temperatures $T_{0}<M_{f}$ and $T_{1}>A_{f}$; the figure clearly shows that the hysteretic behavior of the material is properly described in the range of temperatures between $M_{f}$ and $A_{f}$, and the dead zone of transformation, when the temperature exceeds $A_{f}$ or falls below $M_{f}$, are also simulated.

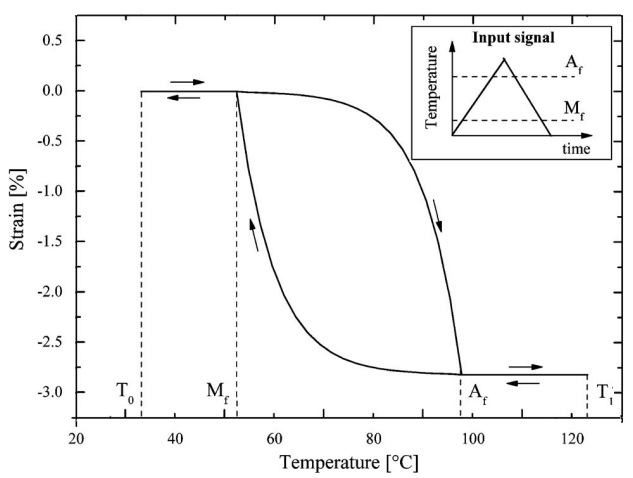

a)

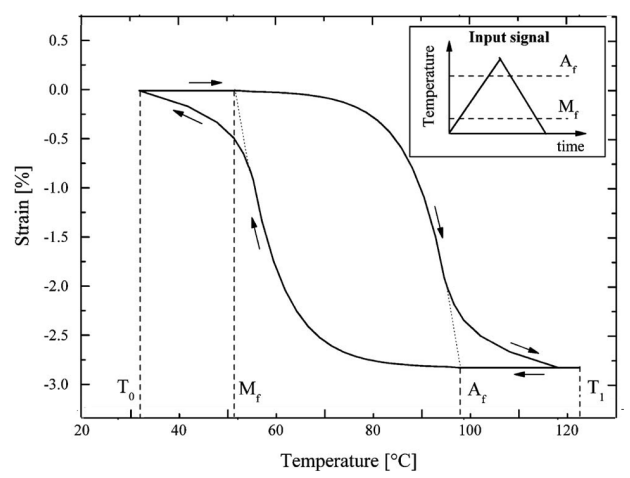

b)

Figure 25. Numerically simulated loop for a thermal cycle between the temperatures $T_{0}<M_{f}$ and $T_{1}>A_{f}$ obtained by: a) Prandtl-Ishlinskii model and b) modified Prandtl-Ishlinskii model [28].

Unfortunately, when comparing the experimental results with the numerically simulated loops, high errors are observed in the extremity of the hysteretic region, i.e. when the temperature is below $M_{f}$ during cooling and above $A_{f}$ during heating. To overcome this limitation, a modified Simulink model can be implemented by including the submodel \#2 of Figure 24. This latter uses two subsystems, for the heating and cooling branches of the loop, which modifies the output response of the system when the temperature is near $M_{f}$ and $A_{f}$. In particular, each subsystem implements a weighted superposition of several dead band operators, which is executed by a series of a dead band block and a gain block, while the saturation block assures that the correction is carried out only in a limited range of temperatures near $M_{f}$ and $A_{f}$. Figure 25.b shows a simulated hysteresis loop, obtained by the modified model, between the temperatures $T_{0}<M_{f}$ and $T_{1}>A_{f}$; the figure clearly shows that the model allows a better simulation of the extremity of the hysteretic region with respect to Figure 25.a.

\subsubsection{Numerical results vs experiments}

In this section, the accuracy and efficiency of the 1D numerical model are illustrated by comparing some experimentally measured hysteresis loops with the corresponding numerical predictions. The simulations have been carried out by using a model with 20 backlash operators and 5 dead zone operators to modify the loops in the extremity of the hysteretic region. Figure 26.a shows a comparison between the experimentally measured hysteresis loop 
for a stress-free martensitic transformation, between the temperatures $T_{0}<M_{f}$ and $T_{1}>A_{f}$, and the numerically simulated one; the figure clearly shows a good accuracy of the numerical model with very small errors. In Figure 26.b, a comparison between numerical predictions and experimental results when the material is subjected to a tensile stress $\sigma=100 \mathrm{MPa}$ is shown. Also, in this case a satisfactory agreement is observed, but the errors increase with respect to the stress free condition. However, it is important to point out that the model parameters were identified by using the measured hysteresis loop under stress-free conditions, and by applying Equations 1 and 11 to modify both the PTTs and $\varepsilon_{t w}$.

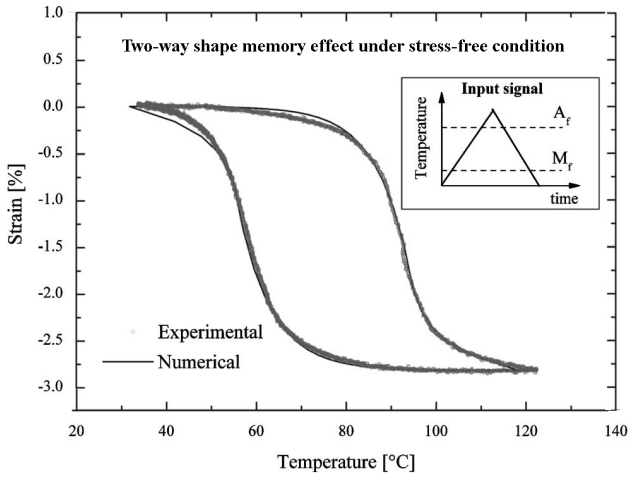

a)

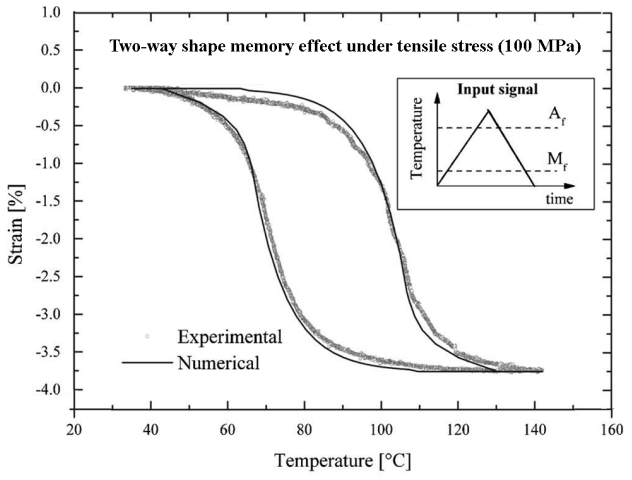

b)

Figure 26. Comparison between experimental measurements and numerical predictions for a thermal cycle between the temperatures $T_{0}<M_{f}$ and $T_{1}>A_{f}$ under a) stress-free condition and b) tensile strees of $100 \mathrm{MPa}$ [28].

The accuracy of the model was also analyzed when the material is subjected to partial thermal cycles, i.e. to incomplete martensitic transformations. Figures 27.a and 27.b show the hysteretic behavior of the material for two different temperature-time paths in stress-free conditions; in particular, Figure 27.a shows incomplete $A \rightarrow M$ transformations, while Figure
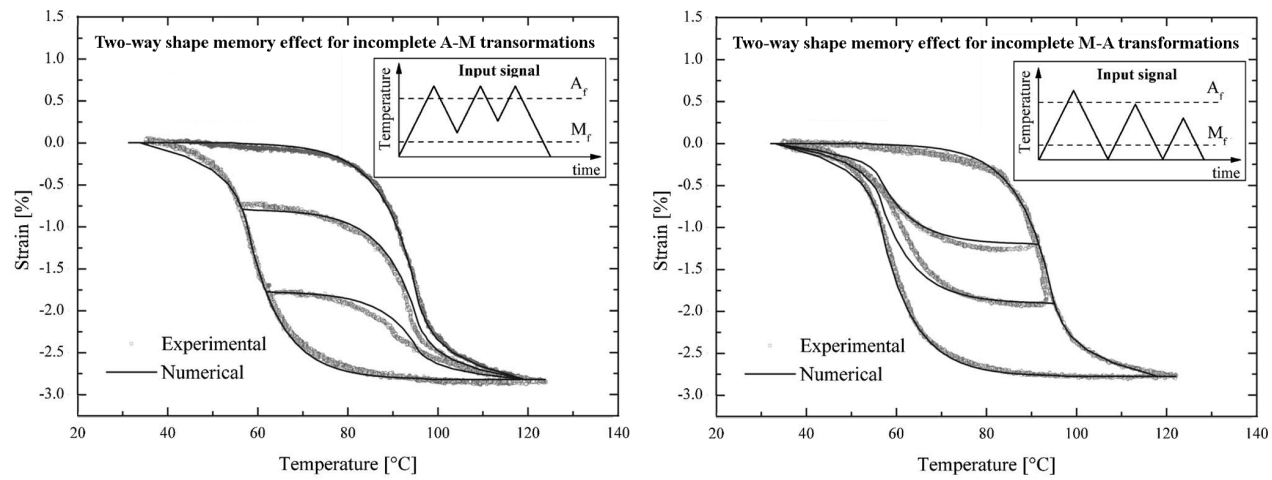

Figure 27. Comparison between experimental measurements and numerical predictions for two different temperature-time paths: a) incomplete $A \rightarrow M$ transformations and b) incomplete $M \rightarrow A$ transformations [28]. 
27.b illustrates incomplete $M \rightarrow A$ transformations. As shown in the figures, the comparison between experimental measurements and numerical predictions show a good accordance in both cases. It is worth noting that the same model parameters of the first example (Figure 26.a) were used in these numerical simulations.

\section{Author details}

Maletta Carmine

Assistant professor of Machine Design at University of Calabria, Department of Mechanical Engineering, Rende (CS), Italy

Furgiuele Franco

Full professor of Machine Design at University of Calabria, Department of Mechanical Engineering, Rende (CS), Italy

\section{References}

[1] Otsuka K, Ren X, (2005) Physical Metallurgy of TiĐNi-Based Shape Memory Alloys. Prog. Mater. Sci. 50: 511-678.

[2] Otsuka K, Wayman CM (1998) Shape Memory Materials. Cambridge University 559 Press, Cambridge.

[3] Wu Ming H (2002) Fabrication of Nitilon Materials and Components. Mater. Sci. Forum. 394-395:285-292.

[4] Schlossmacher P, Haas T, Shussler A (1997) Laser-Welding of a Ni-Rich Tini Shape Memory Alloy: Mechanical Behavior. J. Phys. IV. 7(5):251-256.

[5] Tuissi A, Besseghini S, Ranucci T, Squatrito F, Pozzi M (1999) Effect of Nd-YAG Laser Welding on the Functional Properties of the Ni-49.6 at. \%Ti. Mater. Sci. Eng. A. 273-275:813-817.

[6] Theisen W, Schuermann A (2004) Electro Discharge Machining of Nickel-Titanium Shape Memory Alloys. Mater. Sci. Eng. A. 378:200-204.

[7] Falvo A, Maletta C, Furgiuele F M (2005) Laser Welding of a Niti Alloy: Mechanical and Shape Memory Behavior, Mater. Sci. Eng. A. 412:235-240.

[8] Falvo A, Maletta C, Furgiuele F M (2006) Functional Behavior of a Niti Welded Joint: Two-Way Shape Memory Effect. Mater. Sci. Eng. A. 481-482:647-650.

[9] Gall K A, Seitoglu H, Chumlyakov Y (2000) Niti Experiments Versus Modeling: Where Do We Stand?, Proc. SPIE. 3992:536Đ547.

[10] Paiva A, Savi M A (2006) An Overview of Constitutive Models for Shape Memory Alloys. Math. Probl. Eng. art. no. 56876.

[11] Falk F (1980) Model Free-Energy, Mechanics and Thermodynamics of Shape Memory Alloys. Acta Metall. 28(12):1773-1780.

[12] Falk F (1983) One-Dimensional Model of Shape Memory Alloys. Arch. Mech. 35(1):63-84.

[13] Tanaka K, Nagaki S (1982) Thermomechanical Description of Materials With Internal Variables in the Process of Phase Transitions. Ing. Arch. 51:287-299.

[14] Liang C, Rogers C A (1990) One-Dimensional Thermomechanical Constitutive Relations for Shape Memory Materials. J. Intell. Mater. Syst. Struct. 1:207-234. 
[15] Brinson L C (1993) One Dimensional Constitutive Behavior of Shape Memory Alloys: Themomechanical Derivation With Non-Constant Material Functions and Redefined Martensite Internal Variable. J. Intell. Mater. Syst. Struct. 4:229-242.

[16] Bertran A (1982) Thermomechanical Constitutive Equations for the Description of Shape Memory Effects in Alloys. Nucl. Eng. Des. 74(2):173-182.

[17] Souza A C, Mamiya E, Zouain N (1998) Three-Dimensional Model for Solids Undergoing Stressinduced Phase Transformations. Eur. J. Mech. A/Solids. 17(5):789-806.

[18] Boydand J G, Lagoudas D C (1996) A Thermodynamical Constitutive Model for Shape Memory Materials. Part I. The Monolithic Shape Memory Alloy International Journal of Plasticity. 12(6):805-842.

[19] Auricchio F, Lubliner J (1997) A Uniaxial Model for Shape Memory Alloys. Int. J. Solids Struct. 34(27):3601-3618.

[20] Auricchio F, Sacco E (1997) A One-Dimensional Model for Superelastic Shape Memory Alloys With Different Elastic Properties Between Austenite and Martensite. Int. J. Non-Linear Mech. 32(6):1101-1114.

[21] Auricchio F, Taylor R L, Lubliner J (1997) Shape-Memory Alloys: Macro and Numerical Simulations of the Superelastic Behavior. Comput. Methods Appl. Mech. Eng. 146(3-4): 281-312.

[22] Marfia S, Sacco E, Reddy J N (2003) Superelastic and Shape Memory Effects in Laminated Shape-Memory-Alloy Beams. AIAA J. 41:100-109.

[23] Liew K M, Ren J Reddy J N (2005) Numerical simulation of thermomechanical behaviors of shape memory alloys via a non-linearmesh-free Galerkin formulation. Int. J. Numer. Meth. Engng 63:1014-1040.

[24] Amalraj J, Bhattacharyya A, Faulkner M G (2000) Finite element modeling of phase transformation in shape memory alloy wires with variable material properties. Smart Mater. Struct. 9:622-31.

[25] Krejci P, Kuhnen K, (2001) Inverse Control of Systems With Hysteresis and Creep, IEE Proc.: Control Theory Appl. 148(3):185-192.

[26] Kuhnen K, Janocha H (2001) Inverse Feedforward Controller for Complex Hysteretic Nonlinearities in Smart Material Systems. Control Intell. Syst. 29(3):74-83.

[27] Falvo A, Maletta C, Furgiuele F M (2007) Two-Way Shape Memory Effect of a Ti Rich Niti Alloy: Experimental Measurements and Numerical Simulations. Smart Mater. Struct. 16:771-778.

[28] Falvo A, Maletta C, Furgiuele F M (2008) A Phenomenological Approach for Real-Time Simulation of the Two-Way Shape Memory Effect in NiTi Alloys. ASME Journal of Engineering Materials and Technology. 130:771-778.

[29] Falvo A, Maletta C, Furgiuele F (2008) Hysteresis modeling of two-way shape memory effect in NiTi alloys. Meccanica. 43:165-172.

[30] Falvo A, Maletta C, Furgiuele F (2009) A phenomenological model for superelasticity in NiTi alloys. Smart Mater. Struct. 18:025005.

[31] Raniecki B, Lexcellent C H and Tanaka K 1992 Thermodynamic Models of Pseudoelastic Behavior of Shape Memory Alloys .Arch. Mech. 44 261-284.

[32] Hamilton R F, Sehitoglu H, Chumlyakov Y, Maier H J (2004) Stress Dependence of the Hysteresis in Single Crystal Niti Alloys. Acta Mater. 52:3383-3402. 
144 Smart Actuation and Sensing Systems - Recent Advances and Future Challenges

[33] Sittner P, Landa M, Lukas P, Novak V (2006) R-phase transformation phenomena in thermomechanically loaded NiTi polycrystals. Mechanics of Materials 38:475-492 